\title{
Detection of Mycobacterium Tuberculosis in the Saliva of Patients having Pulmonary Tuberculosis
}

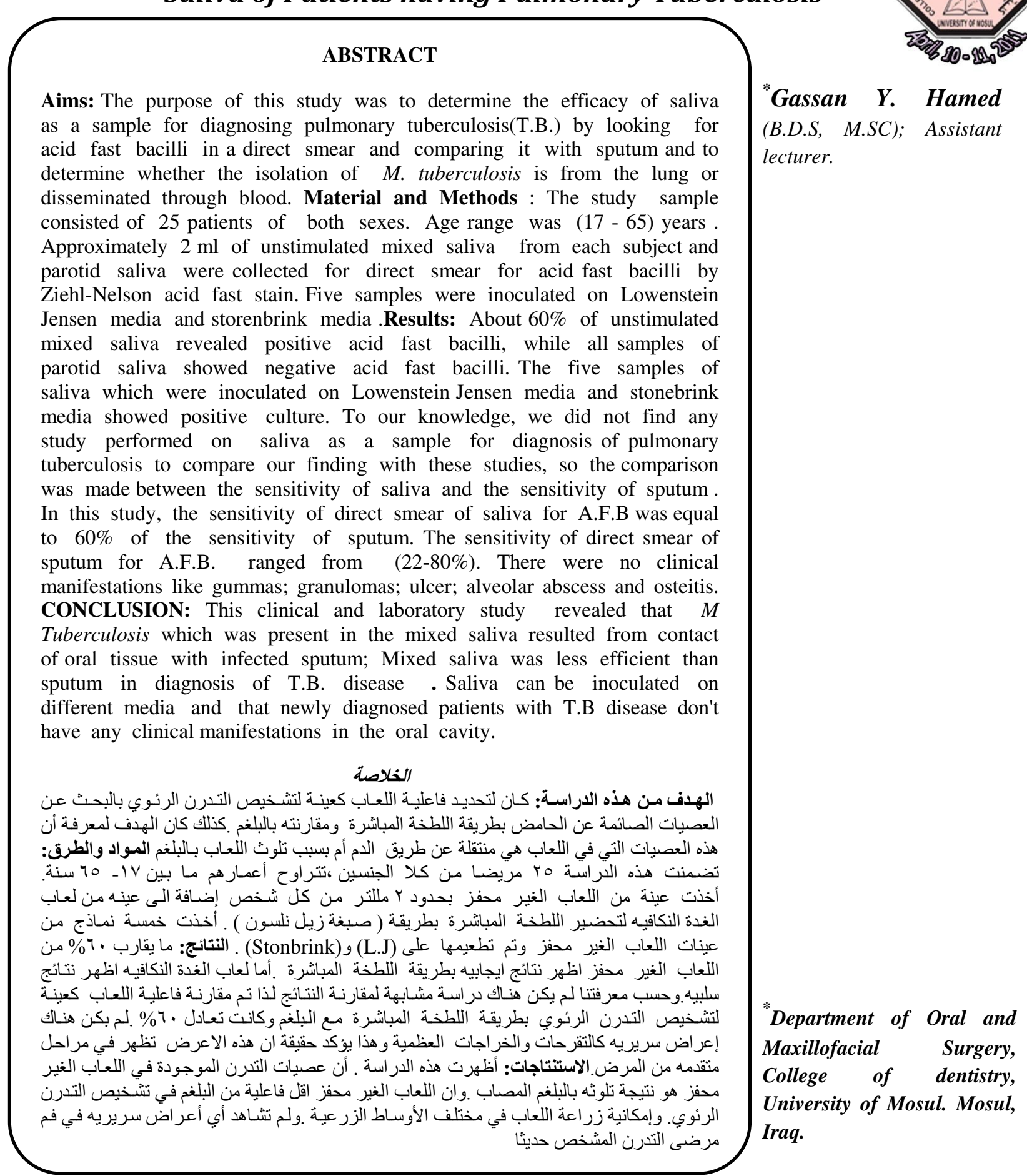

Key Words: M. Tuberculosis, saliva, pulmonary tuberculosis. 


\section{INTRODUCTION}

uberculosis is a chronic infectious disease caused by $M$
tuberculosis. Worldwide, more than 1 billion are infected, with 8 million new
cases and 3 million deaths per year ${ }^{(1)}$. The spread of $M$. tuberculosis infection is
through small airborne droplets, which carry the organism to pulmonary air spaces. Phagocytosis by alveolar macrophages follows, and the battle between bacterial virulence and host resistance begins. The pathogenicity of $M$. tuberculosis is due both to its ability to resist degradation by macrophages and to the development of a type IV hypersensitivity reaction. This latter feature explains the destructiveness of the lesions in the host tissues and the emergence of drug resistant strains ${ }^{(2)}$. The variable nature of its manifestation, as well as its ability to involve almost every organ system, either singly or multiply, makes it essential that the possibility of extra pulmonary tuberculosis be included in the differential diagnosis of any infectious process in the body ${ }^{(3)}$. Once symptoms become apparent, they usually are nonspecific and could be associated with any infectious disease. They include cough, lassitude and malaise, anorexia , unexplained weight loss, night sweats , and fever ${ }^{(4)}$. Oral manifestations may occur in up to $3 \%$ of patients with long-term systemic T. ${ }^{(5)}$. The purpose of this study was to determine the efficacy of saliva as a sample for diagnosing pulmonary tuberculosis(T.B.) by looking up for acid fast bacilli in direct smear and comparing it with sputum. Also to determine whether the isolation of $M$. tuberculosis is from the lung or disseminated through blood.

The purpose of this study was to determine the efficacy of saliva as a sample for diagnosing pulmonary tuberculosis (T.B.) by looking up for acid fast bacilli in direct smear and comparing it with sputum and to determine whether the isolation of $M$. tuberculosis is from the lung or disseminated through blood.

\section{Patients:}

\section{Materials and Methods}

This study included 25 individuals of both sexes who were diagnosed of being having pulmonary tuberculosis. Their ages ranged from (17-65) years old with no signs and symptoms of systemic disease other than TB. Those patients were selected from Chest and Respiratory Disease Institute, TB Lab. Reference in Baghdad between (2001- 2002).

\section{Method of sample collection:}

Sample of unstimulated mixed saliva were obtained under a standard condition. The saliva specimens were collected between 10-12 a.m. at least 2 hours after eating or any oral hygiene procedure. They were instructed to spit the accumulated saliva periodically into a sterile cup. Another sample of parotid saliva was collected by drying and locating the orifice of the parotid duct and collecting the sample of saliva by a blunt instrument for direct smear to detect tuberculous bacilli.

\section{Materials:}

The materials used for the study included:

\section{Lowenstein Jensen (basis)}

\section{Stone bring media}

3. Ziehl -Nelson stain

\section{Methods of examination:}

1. A questionnaire form was designed for this study which included: Age, sex, occupation and medical histories. 


\section{Gassan YH.}

2. Oral examination: The oral mucosa was examined for any clinical manifestation like gummas; granulomas; ulcer; alveolar abscess and osteitis.

3. Specimen collection and processing:

\section{Z-N acid fast stain:}

Diagnosis of tuberculosis is usually achieved by demonstration of acid-fast bacilli (AFB) in expectorated salaiva, The organism have cell wall with high lipid conten special staining procedures are used to prompt the uptake of dye, and once stained, mycobacteria are not easily decolorized even with acid-alcohol. This resistance to decolorization by acid-alcohol is termed acid-fastness. Heating the slide allows greater penetration of carbol fuchsin into the cell wall, mycolic acid and waxes complex the basic dye, which then fails to wash out with acid decolorization ${ }^{(6)}$.

Method : The steps of staining by Z-N stain

1. Heat- Fix slides on a hot plate.

2. Flood smear with carbol fuchsin stain reagent and steam the slides gently for 1 minute. This can be accomplished by flaming from below the rack with a gas burner, or by staining the slides directly on a special hot plate. Do not permit the slides to boil or dry out.

3. Allow the stain to remain on the slides for an additional 4-5 minutes without heat.

4. Rinse with deionized water and tilt slides to drain

5. Decolorize with acid alcohol (95\% ethanol and 3\% hydrochloric acid) for 3 minutes.

6 . Rinse slides with deionized water and tilt to drain.

7. Flood slides with methylene blue reagent for 1 minute.

8. Rinse with deionized water and allow to air dry.

9. Examine under oil immersion (1000x) for presence of acid-fast bacilli.

\section{Culture of mycobacterium:}

Commonly used digestion-decontamination methods which was described with step by step instruction in the guide by Kent and Kubica. In general, the specimen was diluted with an equal volume of digestant and allowed to incubate. A neutralizing buffer is added and the specimen is centrifuged to sediment any AFB present. The centrifugation should be carried out at $\geq 3000 \mathrm{xg}$ for $15 \mathrm{~min}$. To get maximum recovery; some laboratories recommend $3.800 \mathrm{xg}$. The sediment is then inoculated onto the appropriate liquid and solid media.

\section{Statistical analysis:}

Statistical computations were calculated using SPSS 10 for windows software (SPSS Inc, Chicago, IL, USA)on Pentium 4 computer. Statistical significance assessed at the $\mathrm{P} \leq 0.05$ level.

\section{RESULTS}

Twenty-five patients were submitted for investigation. Those patients were diagnosed as Tuberculosis patients by isolating M. Tuberculosis from the sputum. M. tuberculosis was isolated from the oral cavity by 2 ways:

1. Using unstimulated mixed saliva. By this way, 15 patients $(60 \%)$ revealed positive acid fast bacilli while, 10 patients (40\%) showed negative acid fast bacilli.(Table 1).

2. Using parotid saliva. All samples showed negative results(Table 2).

The duration of sign and symptoms of tuberculosis for these 25 patients ranged from (112) months. In order to compare the duration of signs and symptoms with the result of direct 


\section{Gassan YH.}

smear of saliva we divided the duration into 4 groups. Highest found in patients with pulmonary TB of duration (7-9) months. There was no significant relationship between the duration of signs and symptoms of disease with the presence of M. Tuberculosis. (Table $3)$.

Table (1) : Comparison between the sputum and unstimulated mixed saliva in regard to sensitivity for A.F.B

\begin{tabular}{lccccc}
\hline Sample & + & $\%$ & - & $\%$ & Total \\
\hline Mixed saliva & 15 & 60 & 10 & 40 & 25 \\
Sputum & 25 & 100 & 0 & 0 & 25 \\
\hline
\end{tabular}

Table (2) : Comparison between the sputum and two type of saliva in regard to sensitivity for

\begin{tabular}{llll}
\multicolumn{4}{c}{ A.F.B } \\
\hline Sample & + & - & Total \\
\hline Mixed saliva & 15 & 10 & 25 \\
Parotid saliva & 0 & 25 & 25 \\
Sputum & 25 & 0 & 25 \\
\hline
\end{tabular}

Table (3) : Comparison between the duration of signs and symptoms and the results of direct smear of unstimulated mixed saliva

\begin{tabular}{lllll}
\hline $\begin{array}{c}\text { Duration of Signs } \\
\text { and Symptoms }\end{array}$ & + & - & Total \\
\hline $1-3$ & month & 12 & 5 & 17 \\
$4-6$ & month & 2 & 2 & 4 \\
$7-9$ & month & 0 & 1 & 1 \\
$10-12$ & month & 1 & 2 & 3 \\
\hline \multicolumn{5}{c}{$\mathrm{X}^{2}=3.350, \mathrm{df}=3, \mathrm{p}=0.341$}
\end{tabular}

\section{Cultivation:}

Five samples of mixed saliva were chosen randomly to be inoculated on the Lowenstein Jensen media and stone brink media. This was performed commonly by the use of digestion-decontamination methods as described with step by step instruction in the guide by Kent and Kubica, 1985. All samples showed positive growth of $M$. Tuberculosis on both culture media. 


\section{Oral manifestations:}

The oral mucosa was examined for any clinical manifestation by using artificial lighting with the aid of a plane mouth mirror. There were no clinical manifestations like gummas, granulomas, ulcers, alveolar, abscesses and osteitis.

\section{DISCUSSION}

In this study, 25 patients having pulmonary tuberculosis were diagnosed by direct smear of sputum for A.F.B. By using mixed saliva from those patients with pulmonary tuberculosis, $15(60 \%)$ revealed the presence of M. Tuberculosis in the saliva. This confirms the fact of the presence of M. Tuberculosis in the saliva of patient having pulmonary tuberculosis ${ }^{(4)}$. We were unable to identify M.. Tuberculosis in the parotid saliva. This means that M. Tuberculosis which was identified in the mixed saliva was not present in the saliva primarily but it resulted from contact of the oral tissue with infected sputum. Up to our knowledge we did not find any study performed on the saliva as a sample for diagnosis of T.B to compare our finding with these studies, so we did our comparism between the sensitivity of saliva and the sensitivity of sputum. In this study the sensitivity of direct smear of saliva for A.F.B. equals to $60 \%$ of the sensitivity of sputum. The sensitivity of direct smear of sputum for A.F.B. ranged from $(22-80 \%){ }^{(7,8)}$. The density of $M$. Tuberculosis in the mixed saliva mainly is scanty which mean it is not more than (2-9) bacilli in at least (100) field. This is to confirm the fact that the body fluid commonly contains only a small number of $M$. Tuberculosis ${ }^{(9)}$. This means that mixed saliva is less efficient than sputum because by direct smear of sputum the quantity of A.F.B observed on the smear can be provided which serves in the demonstration of the severity of disease, to overcome this shortage, it seems necessary to concentrate the sample of saliva by using, cytocenterfugation ${ }^{(10)}$, or sequential layering of several drops of uncenterfuged fluid on slide, or polycarbonate membrane ${ }^{(9)}$.

\section{Cultivation:}

To explain the ability of using saliva as a sample for culture, five samples of mixed saliva were selected randomly to be inoculated on the Lowenstein Jensen media and stone brink media. All samples revealed positive culture. This means that the $M$. Tuberculosis which was recovered well by Lowenstein Jensen media and M. Bovis which was recovered by stone brink media are present in the saliva ${ }^{(11)}$. This difference between sample of saliva and sputum is shown in (Table 4).

\section{Oral manifestation :}

There were no clinical manifestations like gummas; granulomas; ulcer;alveolar abscess and osteitis. This confirms the fact that these clinical manifestations often develop in patients with active, usually long standing pulmonary tuberculosis and observed more often in patient with advanced disease. The explanation of these findings may be due to the following, all patient of this group are with a short duration of disease, the mechanical cleansing action of saliva and food, the thickened oral epithelium when intact also may minimize the frequency with which lesion develop in these tissues ${ }^{(12)}$. 


\section{Gassan YH.}

Table (4) Comparison between the saliva and sputum

\begin{tabular}{l}
\hline Saliva \\
\hline Always available \\
Less efficient than sputum because is \\
like other body fluids commonly \\
contain only small numbers of \\
Mycobacteria \\
Can be concentrated to maximize the Also can be concentrated to maximize \\
yield of Mycobacteria before the yield of Mycobacteria before \\
inoculation on media and direct smear inoculation of media and direct smear \\
Can be inoculated to liquid and solid Can be inoculated to liquid and solid \\
media \\
The sample which revealed positive is The sample which revealed positive \\
always scanty so we cannot graduate \\
the severity of disease \\
may be scanty or moderate or severe \\
so can measure the severity of \\
disease
\end{tabular}

\section{CONCLUSION}

Conclusions derived from this study are : Mycobacterium Tuberculosis which is present in the mixed saliva results from contact of oral tissue with infected sputum. Mixed saliva is less efficient than sputum in diagnosis of T.B disease. Saliva can be inoculated on different media and we can use it if the sputum is unavailable. The newly diagnosed patients with T.B disease don't have any clinical manifestation in the oral cavity.

\section{REFERENCES}

1. Brad W, Douclas D,Carl M,Jerry E. Oral and Maxillofacial Pathology.2ed. W.B Saunders company.2002, P 173.

2. Joseph A, James J, Richard C. Oral Pathology $5^{\text {th }}$ ed.Linda Duncan.2008, P 161.

3. Mathew R, George F. Extrapulmonary tuberculosis experience of a community hospital and review of the literature. Am J Med. 1985,467-477.

4. James W, little Donald A , Craig S, Miller, Nelson L, Rhodus. Pulmonary disease, In: Dental management of the medically compromised patient, $7^{\text {th }}$ Edition. 2008, p 116,117.

5. Malcolm A, Lynch, Diseases of the respiratory system. In : Burkitt's oral medicine diagnosis and treatment, $11^{\text {th }}$ Edition. BC Decker Inc. 2008, p 488.

6. Eugen W,Denise G,Nancy N,Martha T. Microbiology Ahuman perspective. Mc Graw -Hill. 2004,P47.

7. Lipsk BJ, Gates FC, Tenove JJ. Factors affecting the clinical value of microscopy for acid fast bacilli. Revi Infec Dis. $1984 ; 214-222$.

8. Murray PR, Elmore, Krogsted. The acid fast stain : a specific \& predictive test for mycobacterial disease. Ann Intern Med 1980; 92 : 512-513.

9. Beverly G, Metchock, Fredricks, Richardi JR . Mycobacterium; In : Manual of clinical microbiology, $7^{\text {th }}$ Edition., ASM press Washingotn DC. 1998,P:399-437.

10. Saceanu C N, Pfeiffer, Mclean T. Evaluation of sputum smear concentrated by cytocentrifugation for detection of acid fast bacilli. J Clin Microbiol. 1998; 2371- 2374 . "Cited by Patrick R. Murray" .

11. Zaher F, Marks J. Methods and medium for culture of tubercle bacilli. Tubercle. 1997; 143-145.

12. Malcolm A, Lynch J, Martin S.Oral medicine in :Disease of the respirotary system.JB.Lippincott. 1994,P444. 